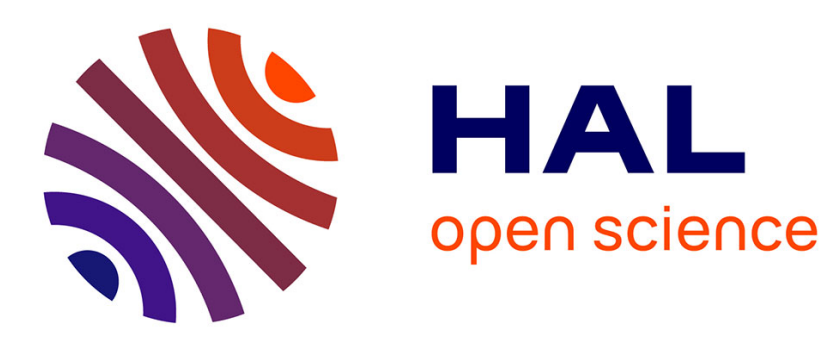

\title{
Pulsation rapide d'un faisceau d'accélérateur au moyen d'une déflexion à onde progressive
}

\author{
M. Roche
}

\section{To cite this version:}

M. Roche. Pulsation rapide d'un faisceau d'accélérateur au moyen d'une déflexion à onde progressive. Revue de Physique Appliquée, 1969, 4 (2), pp.159-160. 10.1051/rphysap:0196900402015901 . jpa00243195

\section{HAL Id: jpa-00243195 https://hal.science/jpa-00243195}

Submitted on 1 Jan 1969

HAL is a multi-disciplinary open access archive for the deposit and dissemination of scientific research documents, whether they are published or not. The documents may come from teaching and research institutions in France or abroad, or from public or private research centers.
L'archive ouverte pluridisciplinaire HAL, est destinée au dépôt et à la diffusion de documents scientifiques de niveau recherche, publiés ou non, émanant des établissements d'enseignement et de recherche français ou étrangers, des laboratoires publics ou privés. 


\title{
PULSATION RAPIDE D'UN FAISGEAU D'AGGÉLÉRATEUR AU MOYEN D'UNE DÉFLEXION A ONDE PROGRESSIVE
}

\author{
M. ROCHE, \\ C.E.A., Centre d'Études de Valduc.
}

Résumé. - On décrit un dispositif destiné à produire des impulsions neutroniques de forme rectangulaire à fronts très brefs $\left(1,4 \times 10^{-9} \mathrm{~s}\right)$.

Abstract. - We describe a device which produces rectangular neutron pulses with a very steep wavefront $\left(1.4 \times 10^{-9} \mathrm{~s}\right)$.

Dans nos expériences de mesure des paramètres cinétiques du réacteur rapide Rachel, il était nécessaire, pour étudier la décroissance transitoire consécutive à une excitation impulsionnelle du réacteur (dont la durée est de l'ordre de $10^{-8} \mathrm{~s}$ ), de disposer de bouffées neutroniques ayant une allure rectangulaire et des fronts très brefs dont la durée devait rester voisine de $10^{-9} \mathrm{~s}$. Ce résultat a été obtenu en modulant, par déflexion et hachage, le faisceau d'un petit accélérateur Sames de $150 \mathrm{kV}$. Les neutrons sont obtenus après interaction des deutérons du faisceau, sur une cible classique, au titane tritié.
Dans les premières expériences, on se contentait de défléchir sinusoïdalement le faisceau au moyen d'un champ électrique transversal $\mathrm{HF}$, de façon à le hacher grâce à un diaphragme en forme de fente disposé sur sa trajectoire. Les nombreuses limitations de ce procédé (mauvaise définition de la forme de l'impulsion de courant cible, manque de souplesse d'emploi, instabilité, etc.) nous ont conduits à adopter une déflexion par impulsion rectangulaire.

Les fronts très brefs que nécessitaient nos expériences ne permettaient pas l'utilisation d'un déflecteur classique constitué de deux plaques parallèles. En effet, 
le temps de transit dans le déflecteur est important et tout se passe comme si le signal rapide de déflexion était intégré. Pour éviter cet inconvénient, nous avons adopté une solution consistant à défléchir le faisceau au moyen d'une « onde progressive ». Ainsi le champ électrique transversal de déflexion se propage dans le même sens et a la même vitesse que les particules du faisceau.

Le déflecteur est constitué d'une série de plaquettes de faible longueur $(2 \mathrm{~cm})$ régulièrement distribuées le long de l'axe. Ces plaquettes sont connectées entre elles au moyen de tronçons de câble coaxial de $100 \Omega$, dont la longueur est déterminée de façon à introduire un retard égal au temps mis par le faisceau pour aller du centre d'une plaquette au centre de la suivante. L'onde électrique progresse ainsi par bonds, à la même vitesse que les particules. Cette solution permet d'obtenir un rapport temps de montée sur retard total de la ligne inférieur à ce qu'on peut obtenir avec des lignes à constantes réparties ou localisées classiques, pour autant que les plaquettes présentent une faible capacité parasite avec la masse et que la structure coaxiale soit le moins possible perturbée.

Le hachage du faisceau est obtenu au moyen d'un diaphragme dont l'ouverture en forme de fente centrée sur l'axe du tube est suffisante pour permettre non seulement le passage du faisceau (qui est focalisé dans ce plan), mais également pour absorber d'éventuelles oscillations transversales. En l'absence d'impulsion, la tension de polarisation appliquée sur la ligne dévie le faisceau qui se trouve alors arrêté par le diaphragme. Cette tension de polarisation est convenablement ajus- tée pour qu'en présence d'une impulsion sur le déflecteur le faisceau traverse le diaphragme en son centre.

La faible impédance du câble coaxial utilisé, la nécessité d'appliquer des impulsions haute tension, la fréquence de récurrence élevée et la rapidité des fronts sont autant de spécifications très sévères que le générateur alimentant ce déflecteur doit satisfaire. Nous avons réalisé à cet effet un amplificateur d'impulsions constitué essentiellement en un tube multiplicateur d'électrons dont le courant de sortie alimente sous basse impédance $(75 \Omega)$ la grille d'une tétrode C-1149 adaptée à un fonctionnement en régime impulsionnel, et dont la charge anodique est de $100 \Omega$. Un transformateur d'isolement, à câbles coaxiaux, permet de modifier à volonté le niveau continu des impulsions de sortie comme il est nécessaire pour un fonctionnement convenable de la déflexion.

Les performances de cet amplificateur sont les suivantes : amplitude de l'impulsion de commande, $40 \mathrm{~V}$ sous $50 \Omega$; amplitude de l'impulsion de sortie, $1500 \mathrm{~V}(2000 \mathrm{~V})$; impédance de sortie, $100 \Omega$; durée des fronts, $6 \mathrm{~ns}$; durée de l'impulsion, variable de 5 à $250 \mathrm{~ns}$; fréquence de récurrence, variable entre 0 et $250 \mathrm{kHz}$; rapport cyclique, $10^{-3}$.

Cet appareillage dont la stabilité et la précision se sont révélées excellentes nous a permis de faire des progrès considérables dans la mise en œuvre de la technique des neutrons pulsés. Des bouffées de neutrons ayant une allure bien rectangulaire et des fronts inférieurs à $1,5 \times 10^{-9} \mathrm{~s}$ ont été couramment utilisées dans notre laboratoire. 